\title{
NUMERICAL SIMULATION OF TRANSIENT TEMPERATURE FIELD FOR LASER DIRECT METAL SHAPING
}

\author{
Risheng Long ${ }^{1,2}$, Weijun Liu ${ }^{1}$, Xiaofeng Shang ${ }^{1}$ \\ 'Advanced Manufacture Lab, Shenyang Institute of Automation, CSA, Shenyang, Liaoning \\ Province,China;E-mail: longrisheng@sia.cn. ${ }^{2}$ Graduate School, Chinese Academy of \\ Sciences, Beijing, China.
}

\begin{abstract}
In order to improve the mechanical properties of the parts fabricated by Laser Direct Metal Shaping (LDMS), it is of great significance to understand the distribution regularities of transient temperature field during LDMS process. Based on the "element birth and death" technique of finite element method, a three-dimensional multi-track and multi-layer model for the transient temperature field analysis of LDMS is developed by ANSYS Parametric Design Language (APDL) for the first time. In the fabricated model, Xdirection parallel reciprocating scanning paths is introduced. Using the same process parameters, the results of simulation show good agreement with the microstructure features of samples which fabricated by LDMS.
\end{abstract}

Key words: Laser Direct Metal Shaping, Transient temperature field, Numerical simulation, laser cladding.

\section{INTRODUCTION}

Laser Direct Metal Shaping is a new additive layered manufacturing technology for building components from a computer-aided design model without the use of forming dies, tooling or machining, and it is the further development of rapid prototyping (RP). Compared with conventional manufacturing processes, LDMS has the advantages of lower cost, shorter cycle time, and higher manufacturing precision and flexibility. However, due to highly localized laser heating feature and the cooling effect of substrate, high temperature gradients in Z-direction are produced during LDMS

Please use the following format when citing this chapter:

Long, Risheng, Liu, Weijun, Shang, Xiaofeng, 2006, in International Federation for Information Processing (IFIP), Volume 207, Knowledge Enterprise: Intelligent Strategies In Product Design, Manufacturing, and Management, eds. K. Wang, Kovacs G., Wozny M., Fang M., (Boston: Springer), pp. 786-796. 
process. Not only does it lead to the directional selection of microstructure in the parts fabricated by LDMS, but also results in large thermal stresses in sample and substrate.

Over the past 10 years, due to the complexity of the physical processes involved in LDMS, the research of transient temperature field in LDMS process is quite deficient. Simple mathematical solutions cannot address the practical manufacturing processes, and it is also impossible for any experimental technique to obtain a complete mapping of the temperature distribution in sample. Computational simulation thus plays an indispensable role in the analysis of such process. Especially, with recent advancements in computational power, FEA simulation of transient temperature in LDMS has gradually become feasible. Recently, 3D laser forming simulations are conducted using commercial FEA software or manual programming. $\mathrm{Xi}$ Mingzhe ${ }^{\prime}$, Zhang Yongzhong and Shi Likai developed a 3D single-track FEM model to simulate the transient temperature field in the real process of the metal thin wall fabricated by laser direct deposition. L. Zhanga ${ }^{2}$, E.W. Reutzelb and P.Michalerisc established the minimum requirements for discretization of 3D 3nite element models for laser forming by examining the convergence of the numerical solutions with increased discretization. AnK Kyrsanidi ${ }^{3}$, ThB Kermanidis and SpG Pantelakis carried out numerical and experimental investigation of the laser forming process using finite element method. K. Dai and L. Shaw developed a 3D thermo-mechanical finite element model including the effect of the powder-to-solid transition. H.Hsiehshen ${ }^{5}$ and Lin Jehnming carried out thermal-mechanical analysis on transient deformation during pulsed laser forming.

In the present paper, according to the "element birth and death" technique, a three-dimensional multi-track and multi-layer finite element model for the transient temperature field of LDMS is developed with APDL for the first time. The distribution regularities of transient temperature field during LDMS process are discussed in detail, and the results of simulation are verified by the samples fabricated by LDMS under the same conditions with simulation.

\section{COMPUTATIONAL FORMALTATIONS}

\subsection{Theoretical formulations}

The heat transfer behavior during LDMS process is a complex, nonlinear, transient process and it can be described with the classical heat conduction 
equation in spatial coordinates $(\mathrm{x}, \mathrm{y}, \mathrm{z})$ :

$$
\begin{aligned}
& k_{e 1}\left(\frac{\partial^{2} T}{\partial x^{2}}+\frac{\partial^{2} T}{\partial y^{2}}+\frac{\partial^{2} T}{\partial z^{2}}\right)+k_{e 2}\left(\frac{\partial^{2} T}{\partial x^{2}}+\frac{\partial^{2} T}{\partial y^{2}}+\frac{\partial^{2} T}{\partial z^{2}}\right) \\
& +q=\rho_{1} \cdot c_{1} \frac{\partial T}{\partial t}+\rho_{2} \cdot c_{2} \frac{\partial T}{\partial t}
\end{aligned}
$$

Where $\rho_{1}$ and $\rho_{2}$ are density of the metal powder and substrate, $c_{1}$ and $c_{2}$ are specific of the metal powder and substrate, $k_{e 1}$ and $k_{e 2}$ are the thermal conductivity of the metal powder and substrate, respectively, and $q$ is the total input energy.

Considering the natural convection and radiation, and assuming $k_{e}=k_{e 1}+k_{e 2}, c=c_{1}+c_{2}, \rho=\rho_{1}+\rho_{2}$ express (1) becomes:

$$
\begin{aligned}
& k_{e}\left(\frac{\partial^{2} T}{\partial x^{2}}+\frac{\partial^{2} T}{\partial y^{2}}+\frac{\partial^{2} T}{\partial z^{2}}\right)+q+h\left(T-T_{h}\right)+\sigma \varepsilon\left(T^{4}-T_{h}^{4}\right) \\
& =\rho \cdot c \frac{\partial T}{\partial t}
\end{aligned}
$$

Where $h$ is natural convection exchange coefficient, $\sigma$ is radiation constant of black body, $T_{h}$ is room temperature, $\varepsilon$ is actual radiation coefficient of object.

The equation (2) must be solved with prescribed boundary and initial conditions. The initial conditions can be written as:

$$
T(x, y, z, 0)=T_{h}
$$

On the boundary and the upside surface of the sample, convection and radiation in heat transfer are responsible for heat loss to the ambient. So, the boundary conditions of them can be written as:

$$
-k_{e} \frac{\partial T}{\partial z}=h\left(T-T_{h}\right)+\sigma \varepsilon\left(T^{4}-T_{h}^{4}\right)
$$

The underside of substrate can be treat as adiabatic, and its boundary condition can be written as:

$$
-k_{e} \frac{\partial T}{\partial z}=0
$$




\section{2 "Element birth and death" technique}

"Element birth and death" technique does not actually remove "killed" elements from model. Instead, it deactivates them by multiplying their stiffness or conductivity by a severe reduction factor. This factor is set to $1.0 \mathrm{E}-6$ by default, but can be given other values. Element loads associated with deactivated elements are zeroed out of the load vector, however, they still appear in element-load lists. Similarly, mass, damping, specific heat, and other such effects are set to zero for deactivated elements.

In like manner, when elements are "born", they are not actually added to the model; they are simply reactivated. So, you must create all elements, including those to be born in later stages of your analysis, while in preprocessor. When an element is reactivated, its stiffness, mass, element loads, etc. return to their full original values, but there is no record of strain history (or heat storage, etc.) for it.

\subsection{Heat source and latent heat of phase change}

The heat flux to system is put in by a highly focused area on the molten pool in LDMS process, and it is assumed that the heat flux, $q(r)$, follows Gauss distribution in the radial direction, and has the following form:

$$
q(r)=\frac{3 Q}{\pi R^{2}} \exp \left(-\frac{3 r^{2}}{R^{2}}\right)
$$

Where $\mathrm{R}$ is the laser spot radius, $\mathrm{r}$ is the distance of a point away from the centre of the laser beam, and $Q$ is the total heat input energy.

Simultaneously, phase change, such as meltage and solidification, are existed during LDMS process, and the latent heat of phase change can't be neglected for the crystal materials, such as metal. Phase change problems are usually solved with finite difference or finite element methods, and the most common method used is the enthalpy method.

\section{GEOMETRY CONFIGURE}

To accurately reflect of the characteristics during LDMS process, according to the former theories and "element birth and death" technique, a multi-track and multi-layer FEM model is developed by APDL, in which, Xdirection reciprocating scanning paths are also introduced into the model. 
Fig.1 demonstrates the FEM model for temperature field simulation and the Location of nodes on the longitudinal section. As shown in Fig. 1a, the dimension of substrate is $100 \mathrm{~mm} \times 25 \mathrm{~mm} \times 10 \mathrm{~mm}$, and the size of sample is $50 \mathrm{~mm} \times 4 \mathrm{~mm} \times 6 \mathrm{~mm}$. To decrease the large computational time and still maintain reasonable accuracy, a fine mesh is used in sample which is divided to six layers, each layer $1 \mathrm{~mm}$, and the coarse mesh is adopted in the substrate.

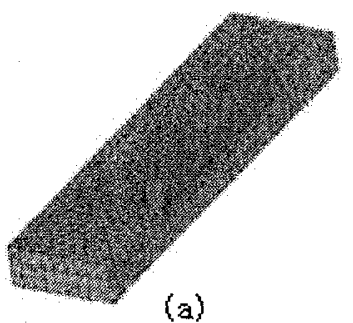

(a)

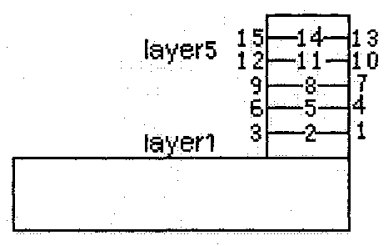

(b)

Figure 1. Schematic of model (a) the FEM model of temperature field, (b) the location of nodes on the longitudinal section.

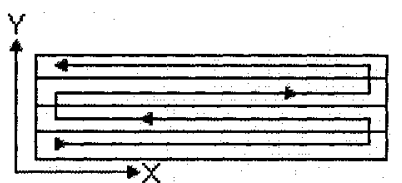

(a)

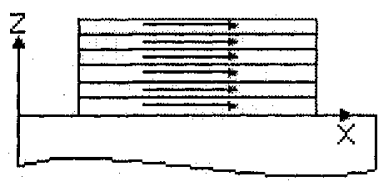

(b)

Figure 2. Schematic of scanning paths in simulation. (a) in XY plane, (b) in XZ plane.

Moreover, Fig. 2 displays the X-direction parallel reciprocating scanning paths, in which Fig.2a and Fig.2b show the schematic of scanning paths in $\mathrm{XY}$ plane and $\mathrm{XZ}$ plane, respectively. As shown in the figure, after one layer is finished, the laser firstly moves from the end of current layer to the start, then lift a little distance along $Z$ direction, which is equal to the height of single layer.

\section{RESULTS AND DISCUSSION}

In simulation, the laser power equals $600 \mathrm{~W}$, spot diameter equals $1 \mathrm{~mm}$, scanning velocity equals $5 \mathrm{~mm} / \mathrm{s}$, room temperature equals $20^{\circ} \mathrm{C}$. The material of metal powder used in simulation is Ni60A, its material properties are 
listed in Table.1. The material of substrate is 45 steel whose material properties are taken from Complex Alloy Steel Manual ${ }^{7}$.

Table 1. Parameters list of Ni60A

\begin{tabular}{ll}
\hline Parameter name & value \\
\hline Specific heat of $\mathrm{Ni60A}\left(\mathrm{J} / \mathrm{mg} \cdot{ }^{\circ} \mathrm{C}\right)$ & 0.000464 \\
Latent heat of $\mathrm{Ni60A}(\mathrm{J} / \mathrm{mg})$ & 0.248 \\
Density of $\mathrm{Ni60A}\left(\mathrm{kg} / \mathrm{m}^{3}\right)$ & 8522 \\
Melting point of $\mathrm{Ni60A}\left({ }^{\circ} \mathrm{C}\right)$ & $950 \sim 1000$ \\
\hline
\end{tabular}

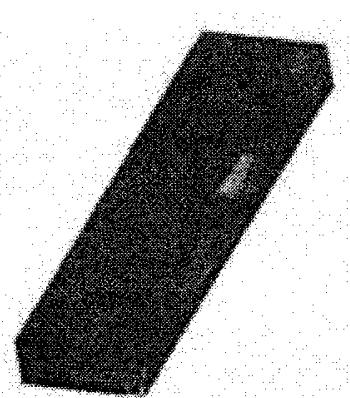

(a)

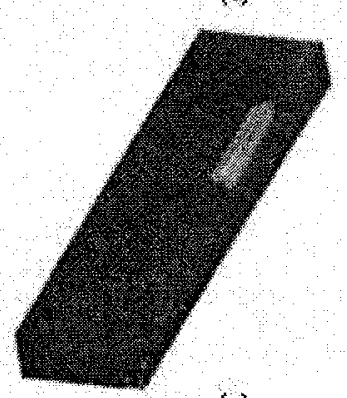

(a)
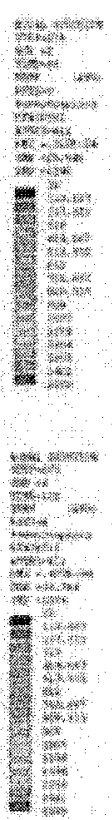

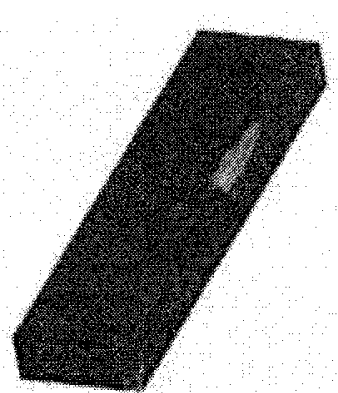

(b)

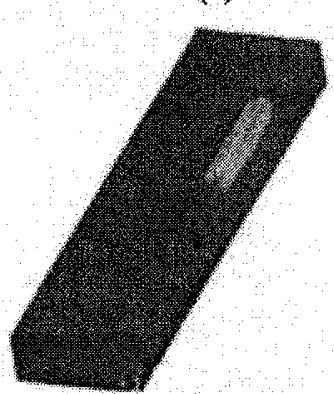

(d)
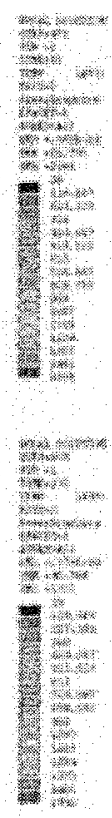

Figure 3. Temperature field contours at different time

Under the condition of Table.1, the temperature field contours of model at different time are demonstrated in Fig.3. As shown in it, Fig.3a shows the contour of temperature field at the 55th second, on this occasion, the second track of the second layer is being processed; Fig. $3 \mathrm{~b}$ shows the contour of temperature field at the 95 th second, on this occasion, the second track of the third layer is being processed; Fig. $3 \mathrm{c}$ shows the contour of temperature field at the 135th second, on this occasion, the second track of the fourth layer is being processed; Fig. $3 \mathrm{~d}$ shows the contour of temperature field at the 175th 
second, on this occasion, the second track of the fifth layer is being processed.

In addition, as seen from Fig.3, the distribution of temperature field in model takes on motion state with the movement of laser heat source, and the high temperature regions enlarge gradually with the increment of layers. The reason is because that the fabricated layers of sample are heated again and again with the increment of layers, the heat accumulation effect existed in them decreases the cooling velocity of the new layer gradually, and it resulted in the highest temperature of molten pool becoming higher and higher.
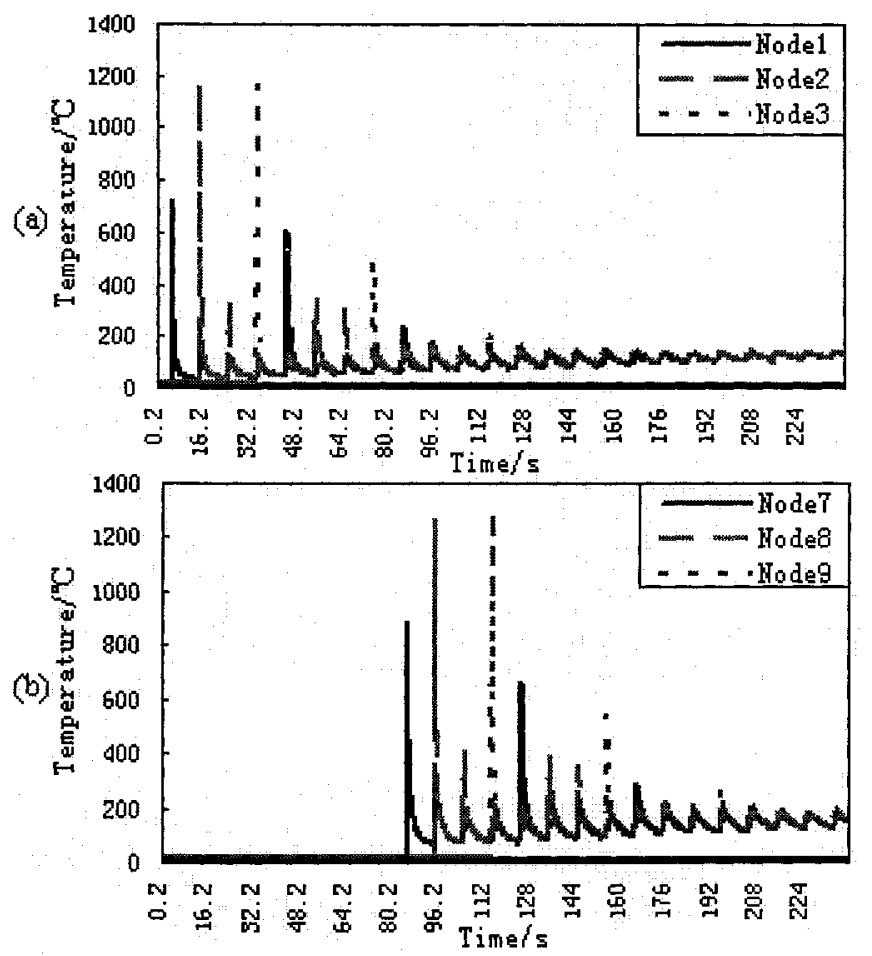

Figure 4. Variations of temperature at locations of different nodes on the longitudinal section. (a) of node 1,2 and 3; (b) of node 7,8 and 9.

Fig.4 displays the variations of temperature at locations of different nodes on the longitudinal section (see Fig.1b). As shown in Fig.4a, which compares the temperature history curves of node 1,2 and 3 in the first layer of sample, the temperature of node 1 is $20^{\circ} \mathrm{C}$ before the 5 th second, just the room temperature that we set, and it rises to $638^{\circ} \mathrm{C}$ quickly at the 5th second. The reason is because node 1 is under "death" condition before the 5th 
second, and there is no temperature variation record for it. But at the 5th second, the laser heat source just moves to the upside of the element which node 1 belonged to, and at the same time the element are reactivated with nodel. Once the nodel is reactivated, the temperature of it rises quickly. In the same way, node 2 is under "death" condition before the 15 th second. But at the 15th second, the laser heat source just moves to the upside of the element which node 2 belonged to, node 2 is reactivated, the temperature of it rises from room temperature to $1168{ }^{\circ} \mathrm{C}$ quickly. Meanwhile, because of the near distance between nodel and node 2 , the temperature variation of node1 will be affected by the heat source synchronously. The temperature variation of node 3 is similar to node 1 and 2 . In the following simulation, node 1, 2 and 3 are heated and cooled down again and again and are tend to the same temperature gradually. The reason is because when the following layer's process moves to the upside of node 1,2 and 3, they will be heated by the energy conducted from molten pool and neighboring areas, but the range of temperature variation of them will become smaller and smaller with the increment of layers.

The temperature variation regularities of the node 7,8 and 9 are similar to the regularities of the node 1,2 and 3 , such as variation period etc., besides the difference of reactivated time, as shown in Fig.4b.

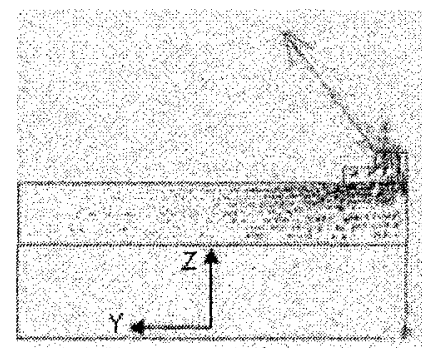

(a)

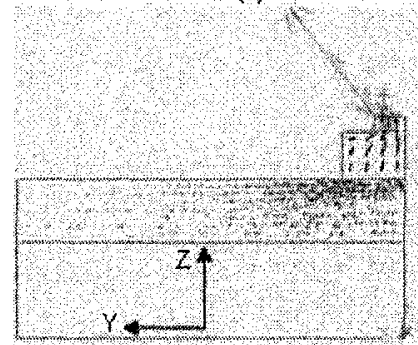

(c)

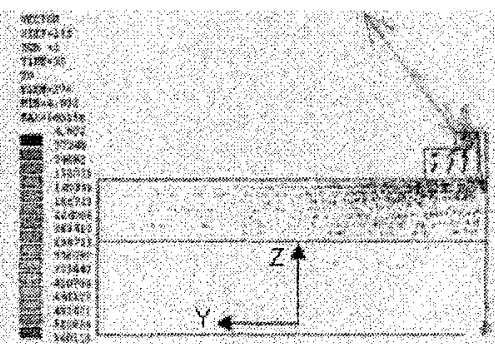

(b)

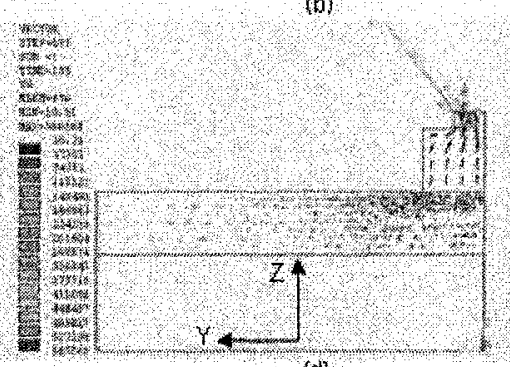

(d)
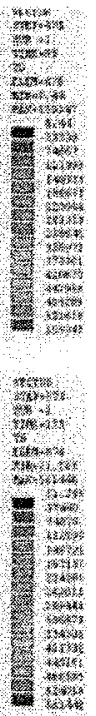

Figure 5. Temperature gradient vector diagrams in simulation at different time. (a) at $55 \mathrm{~s}$, (b) at $95 \mathrm{~s},(\mathrm{c})$ at $135 \mathrm{~s},(\mathrm{~d})$ at $175 \mathrm{~s}$ 
Fig.5 shows the temperature gradient vector diagrams in simulation at different time. From it, there are several features can be gotten and they are listed as the following: (1) The distribution of temperature gradient in sample is non-uniform. It is strong and concentrated at the district which subjected to the laser energy influence, and it is small and dispersive at the district which far away from the laser energy influence. The temperature gradients in molten pool and its nearby district are quite big. (2) The temperature gradient in sample is mainly along the $\mathrm{Z}$ direction, the perpendicular way of substrate, and it is small in other directions. (3) The distribution of temperature gradient in substrate is non-uniform. It is strong and concentrated at the district which near the sample, and it is small and dispersive at the district which far away from the sample. (4) The temperature gradient in substrate is mainly along the direction which parallels the substrate plane, having obvious layering phenomenon.

\section{LDMS EXPERIMENT AND SEM ANALYSIS}

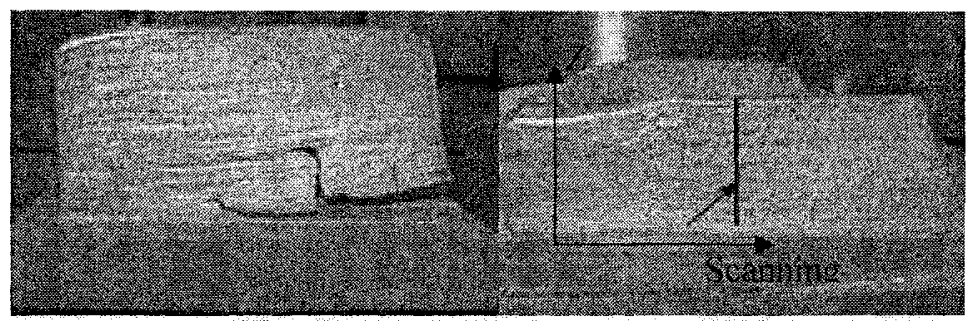

(a)

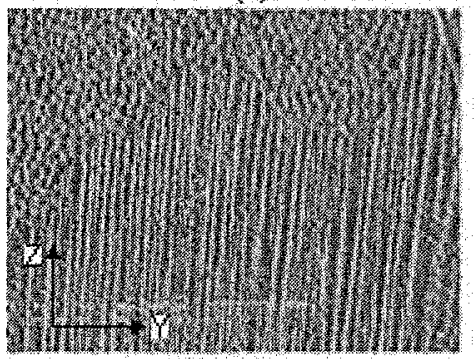

(c) $\times 150$ (b)

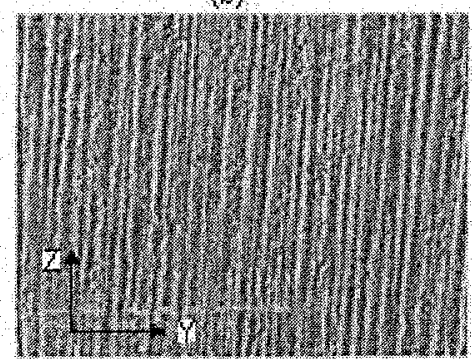

(d) $\times 200$

Figure 6. Photos of the samples fabricated by LDMS and SEM macrographs of the longitudinal section of sample along Z-direction. (in the same cladding layer).(a) at the top of same layer; (b) at the middle of same layer

In the case of $800 \mathrm{~W}$ laser power, $1 \mathrm{~mm}$ spot diameter, $5 \mathrm{~mm} / \mathrm{s}$ scanning velocity, $20^{\circ} \mathrm{Croom}$ temperature, as shown in Fig.6, some nickel-based 
superalloy components are fabricated through the LMDS system which has been developed successfully by Chinese Academy of Sciences Shenyang Institute of Automation (see Fig.6a and Fig.6b). Fig.6c and Fig.6d exhibit the $Z$ direction SEM micrograph of the longitudinal section which marked in Fig.6b.

As shown in Fig.6c and Fig.6d, equiaxed grains appear on the top of the fine dendrites at the top of one layer. Similarly, the solidification microstructure at the middle of same layer is composed of parallel dendrites whose growing direction is nearly parallel to the positive $Z$ direction. The reason is because the temperature gradient in the positive $Z$-direction is remarkably dominant in the process of cooling solidification, and heat mostly dissipates in the negative Z-direction. Therefore, under the action of the highest temperature gradient and solidification rate in the Z-direction, grains grow with the directional selection, thus forming the dendrites which are almost parallel to Z-direction.

\section{CONCLUSIONS}

In this paper, based on the FEM technique of "life and death element", a three-dimensional multi-track and multi-layer model for the transient temperature field analysis of LDMS is developed with APDL for the first time. Through the model, the transient temperature field's distribution regularities are discussed detailedly. Under the same conditions in simulation, the results from simulation show good agreement with the microstructure features of the samples which fabricated by LDMS.

\section{REFERENCES}

1. Xi Mingzhe, Zhang Yongzhong and Shi Likai, (2003), Numerical simulation of 3D transient temperature field in thin-wall metal parts fabricated by laser direct deposition, The Chinese Journal of Nonferrous Metals, vol.13, pp.887 892.

2. L. Zhanga, E.W. Reutzelb and P.Michalerisc, (2004), Finite element modeling discretization requirements for the laser forming process. International Journal of Mechanical Sciences 46, pp.623-637.

3. AnK Kyrsanidi, ThB Kermanidis and SpG Pantelakis, (1999), Numerical and experimental investigation of the laser forming process, Journal of Material Process Technology, vol.872, pp.281 - 290 .

4. K. Dai, L. Shaw, (2004), Thermal and mechanical finite element modeling of laser forming from metal and ceramic powders, Acta Materialia 52, 6980. 
5. H.Hsiehshen, Lin Jehnming, (2004), Thermal-mechanical analysis on transient deformation during pulsed laser forming, International Journal of Machine Tools \& Manufacture, vol.44, pp.191-199.

6. ANSYS Theory Manual, (2003), Release 8.0, ANSYS Inc., USA.

7. Chinese Iron-Steel Research Institue, (1992), Complex Alloy Steel Manual: lower Volume, Metallurgical Industry Press, Beijing, pp.3-45. 